\title{
Eops:
}

\section{Sindrome de piernas inquietas: intervenciones terapéuticas efectivas}

\author{
Restless legs syndrome: effective therapeuic interventions
}

María Cecilia Vallese*

\begin{abstract}
Resumen
Frente a un paciente que consulta por síntomas compatibles con el síndrome de piernas inquietas, la autora del presente artículo revisa brevemente sus criterios diagnósticos así como la disponibilidad de tratamientos farmacológicos basados en la evidencia. Asimismo, se evalúa la relación de este síndrome con los trastornos del sueño. La mayor evidencia hasta el momento la poseen el ropinirol y la pergolida. También se describe junto a otra evidencia de ensayos clínicos aleatorizados de la L-dopa, la carbamazepina, la gabapentina, el hierro, la carbegolina. Poca evidencia sustenta el uso del clonazepam,u otras drogas como la rotigotina, hidroquinona, oxicodona y bromocriptina. Sin embargo, la evidencia para todos los tratamientos disponibles hoy para el SPI es de una manera u otra, algo limitada.
\end{abstract}

Vallese M.C. Síndrome de piernas inquietas: intervenciones terapéuticas efectivas Evid. actual. práct. ambul; 10(4): 124-126, julago.2007.

\section{Escenario clínico}

Una médica de familia atiende a un hombre de 35 años con síndrome de piernas inquietas y movimientos periódicos involuntarios de los miembros durante el sueño de aproximadamente diez años de evolución. Actualmente, consulta por astenia severa y trastornos del sueño que han empeorado en los últimos años. Nunca ha recibido tratamiento por este problema pero le pide ayuda desesperadamente porque "siempre está cansado" y últimamente, ha notado una disminución importante en su desempeño físico. Ha intentado mejorar los síntomas con baños de agua caliente, masajes en los miembros e incluso con tomas esporádicas de clonazepam sin obtener beneficio alguno. No padece ningún cuadro psiquiátrico, no consume alcohol, ni drogas ni presenta comorbilidad asociada.

\section{Pregunta que generó el caso}

En los pacientes con el síndrome de piernas inquietas y movimientos periódicos involuntarios de los miembros (población), ¿existen intervenciones farmacológicas efectivas (intervención) para la reducción de los trastornos del sueño (resultado)?

\section{Estrategia de búsqueda}

Se realiza una búsqueda en la base Cochrane sin encontrar meta-análisis ni revisiones sistemáticas al respecto. Luego, se realiza una búsqueda en MEDLINE por Pubmed empleando como palabras clave "Nocturnal Myoclonus Syndrome" AND "Restless Legs Syndrome". Al intentar buscar meta-análisis o revisiones sistemáticas no se observan resultados, por lo que se volvió a intentar una nueva búsqueda, esta vez en Tripdatabase a través de "Restless Legs syndrome" la que arrojó varias citas interesantes para contestar a la pregunta. Se eligió una revisión sobre las alternativas terapéuticas para este síndrome, realizada por Bandolier, encontrada bajo una de las 8 Preguntas Cínicas que aparecieron por esta búsqueda: "What is the latest evidence for the treatment of restless legs syndrome (RSL)? y que responde en forma muy completa a la pregunta con bibliografía actualizada a marzo de 2005.

\section{SÍNDROME DE LAS PIERNAS INQUIETAS Y SU RELACIÓN CON LOS TRASTORNOS DEL SUEÑO.}

El Síndrome de Piernas Inquietas (SPI) es un trastorno neurológico del movimiento caracterizado por la necesidad irresistible de mover las piernas y por sensaciones molestas y desagradables en las extremidades inferiores. El diagnóstico es eminentemente clínico y se requiere la presencia de cuatro elementos básicos según los últimos criterios establecidos en 2003: ${ }^{1}$

1. Deseo de mover las piernas, generalmente asociado a parestesias y disestesias.

2. Exacerbación de los síntomas con el descanso (cuando se sienta o se acuesta)

3. Tanto el deseo de mover las piernas como las parestesias ceden total o parcialmente con los movimientos (caminar, elongar)

4. Empeoramiento nocturno de los síntomas.

Entre un $80 \%$ y $85 \%$ de las personas con SPI experimentan movimientos involuntarios periódicos de las extremidades durante el sueño. Éstas son sacudidas que se producen habitualmente con una frecuencia de 20 a 30 segundos (entre su aparición y desaparición) a lo largo de la noche, a menudo causando despertares parciales que interrumpen el sueño. Esto afecta, en gran medida, la calidad de vida de los pacientes ya que la privación crónica del sueño afecta de modo negativo la capacidad de concentración y de trabajo, así como la capacidad para conducir, tomar parte en actividades sociales o disfrutar del tiempo libre.

Si bien lo más habitual es que su inicio tenga lugar entre los 40 y 50 años, el SPI puede aparecer en cualquier momento de la vida. Suele manifestarse inicialmente de manera episódica, aumentando su severidad y frecuencia con el tiempo, hasta presentarse prácticamente a diario.

Los resultados obtenidos del estudio $\mathrm{REST}^{2}$ (Estudio de Epidemiología, Síntomas y Tratamiento), el mayor y más amplio estudio realizado en cinco países europeos y Estados Unidos sobre el SPI, concluyen que aproximadamente el $5 \%$ (entre un 10 y 15\%) de la población, padece este síndrome. Entre todos los pacientes que participaron en el estudio, el $81 \%$ había consultado al médico acerca de los síntomas y el $75 \%$ recibió un diagnóstico; aunque a tan sólo el $8,3 \%$ se le realizó un diagnóstico correcto de SPI. En aquellos en los que no se diagnosticó el síndrome, los síntomas que padecían se atribuyeron erróneamente a problemas tales como enfermedades reumáticas o trastornos circulatorios, calambres musculares, nerviosismo o incluso al envejecimiento. Es particularmente frecuente en las mujeres, (hasta dos veces más frecuente que en los hombres) y la probabilidad de padecer esta enfermedad crónica aumenta en función del número de embarazos (hasta tres veces más frecuente a partir del tercer embarazo). Otros factores de riesgo que han sido identificados son la edad (hasta el $15 \%$ en los mayores de 65 años) y las situaciones de déficit de hierro $(30-40 \%$ de las personas con anemia ferropénica). Se suele asociar a enfermedades crónicas como la diabetes, insuficiencia renal $(50 \%$ de las personas en hemodiálisis tiene SPI) o la artritis reumatoide. Sin embar- 
go, la gran mayoría se conoce con el nombre de SPI idiopático debido a que no se puede hallar una causa que lo explique y por ende, su tratamiento actualmente se dirige específicamente a disminuir los síntomas.

Ninguno de los cambios en el estilo de vida propuestos (disminución del consumo de cafeína, alcohol y tabaco, aumento de la actividad física) pudo demostrar científicamente tener algún efecto positivo en la disminución de las molestias ni en los trastornos del sueño secundarios. Tampoco lo lograron medidas simples como los baños con agua caliente, masajes en las piernas, ni aplicarse una bolsa de agua caliente o hielo.

La búsqueda realizada pretendía responder la pregunta sobre la eficacia y/o efectividad de las intervenciones farmacológicas que existen para este trastorno en la actualidad, pasibles de ser utilizadas en atención primaria.

\section{Resumen de la evidencia}

Bandolier ha realizado una serie de revisiones sobre las alternativas terapéuticas para el SPI, evaluando los fármacos estudiados hasta la fecha que presentan al menos un ensayo clínico aleatorizado y controlado (ECCA). ${ }^{3}$

Los estudios en el SPI se caracterizan por ser relativamente muy pequeños y de corta duración, en su mayoría 2 a 4 semanas. Sólo algunos se han realizado por 12 semanas, evaluando al ropinirol. La mayoría son aleatorizados, ciegos y con buena calidad en el reporte de sus resultados.

Los resultados medidos en estos estudios son de distinto tipo: - resultados relacionados con el sueño: éstos son detallados y se han obtenido en relativamente pocos pacientes en laboratorios del sueño. Se han incluido como resultados de este tipo: tiempo de latencia (tiempo hasta la conciliación del sueño), duración del sueño, y algunos aspectos otros más complejos. - Movimientos periódicos de las piernas durante el sueño: generalmente obtenidos en laboratorios de sueño

- Síntomas del SPI: para evaluar los mismos se utilizan en general, un set de síntomas reconocidos internacionalmente, adaptado de los presentados por el Internacional Restless Legs Syndrome Study Group (IRLSSG).

Resultados. El ropinirol y la L-Dopa se estudiaron en 6 ECCA pero fue el ropinirol el que presentó mayor cantidad de datos, fue probado por un mayor horizonte temporal y obtuvo los mejores resultados. La pergolida también tuvo buenos resultados, aunque sólo se probó en 96 pacientes. También se estudiaron otras drogas en pequeños estudios individuales, o en varios, con insuficiente cantidad de información a partir de la cual poder sacar conclusiones satisfactorias.

Las principales conclusiones sobre las diferentes alternativas terapéuticas farmacológicas que se han estudiado en relación con el SPI se resumen a continuación: ${ }^{3}$

L-dopa (6 estudios)4: con esta droga sólo fueron estudiados en forma aleatorizada y controlada 142 pacientes. La mayoría de los estudios son pequeños y se concentraron en parámetros relacionados con las alteraciones del sueño. La L-dopa reduciría los movimientos periódicos de los miembros pero no hay evidencia clara sobre la reducción del resto de los síntomas. Los efectos adversos relacionados con el tratamiento fueron leves. (Evidencia I)

Ropiniro 5 : Se trataron un total de 340 pacientes en 5 ECCA donde compararon el ropinirol con placebo o levodopa; en tres de ellos, los pacientes se siguieron durante 12 semanas. El ropinirol demostró ser efectivo para reducir los síntomas y los movimientos periódicos de los miembros. Todos los estudios demostraron que el ropinirol mejora significativamente las características del sueño. Los puntajes de calidad en estos estudios fueron buenos, pero al haber estado los más grandes financiados por industria farmacéutica, podrían sobrestimar el beneficio. Con respecto a la eficacia, $169 / 310$ pacientes $(55 \%)$ mejoraron bastante o mucho con dosis tituladas de ropinirol comparado con 109/304 (36\%) con placebo o L-dopa luego de 12 semanas de tratamiento. Esto demuestra un RR de 1,5 (1,3 a 1,8$)$ y un NNT de 5,4 (3,9 a 9,2). Hubo más pacientes que experimentaron por lo menos un efecto adverso con ropinirol que con placebo, pero no hubo diferencias en relación a efectos adversos graves ni a la discontinuación del tratamiento debido a los mismos. Entre toda la literatura de estudios clínicos del SPI, este fármaco es el que cuenta con el mayor caudal de pacientes (700), estudios de más larga duración, mejor calidad, resultados más sensibles y con datos suficientes que nos permiten realizar análisis de eficacia y de efectos adversos. (Evidencia I)

Pergolida $^{6}$ : se encontraron 4 ECCA, comparándola con placebo y en algunos casos con L-dopa. Las dosis de pergolida varían entre 0.25 y $0.75 \mathrm{mg}$ diarios, con titulación individual hasta encontrar la dosis de efecto buscado. La duración de los estudios fue hasta 6 semanas. Los resultados evaluados incluyeron síntomas del SPI, estudios del sueño y movimientos periódicos de los miembros. La pergolida se probó en 96 pacientes. En base a la limitada información obtenida a partir de estos estudios, parecería ser efectiva para reducir los síntomas y los movimientos periódicos de los miembros, así como también para mejorar las características del sueño. Luego de 4 a 6 semanas de tratamiento se observó una reducción significativa de los síntomas en el $76 \%$ de los pacientes con pergolida en comparación al $18 \%$ de los pacientes con placebo. El beneficio relativo fue de 4,3 ( 2,8 a 6,6$)$, y el NNT en 4 a 6 semanas es de $1,7(1,4$ a 2,1). No se puede confiar en conclusiones sobre el análisis de los efectos adversos dado el reducido número de pacientes estudiados. (Evidencia I)

Carbamazepina ${ }^{7}$ : esta droga ha sido utilizada solo en un ECCA aleatorizado, doble ciego de 84 pacientes durante 5 semanas, y demostró ser más efectivo que el placebo. En el mismo se comenzó con dosis de $100 \mathrm{mg} /$ día por una semana y se la tituló hasta $300 \mathrm{mg} / \mathrm{día}$ en las semanas 2 y 3 hasta su estabilización en las semanas 4 y 5 . El principal resultado evaluado fue la cantidad de ataques del SPI por semana. Por este estudio, la cabarmazepina ha demostrado ser efectiva para el tratamiento del SPI por lo menos durante un período breve de tiempo. (Evidencia I)

Gabapentina: en base a información muy limitada de 48 pacientes, la gabapentina impresiona ser efectiva en el tratamiento del SPI.

Hierro $^{8}:$ se encontraron dos ECCA pequeños. Uno evaluó 28 pacientes con SPI sin causa secundaria y los trató con sulfato ferroso. El otro evaluó el tratamiento de pacientes con SPI en plan de diálisis con infusión intravenosa de hierro dextrán. EI primer estudio no demostró diferencias significativas para mejorar la calidad del sueño ni los síntomas del síndrome. De todas maneras, la población estudiada fue muy pequeña como para poder sacar conclusiones determinantes sobre estos tipos de tratamiento.

Cabergolina $a^{9}:$ un solo estudio brinda escasa evidencia sobre el valor de esta droga en el tratamiento del SPI. En este estudio, aproximadamente el $45 \%$ de los pacientes tratados con esta droga durante 5 semanas estuvieron sin síntomas (significativamente más importante que en los pacientes tratados con 
placebo) y no hubo diferencias en los eventos adversos entre uno y otro grupo.

Clonazepam: no existe hasta la fecha información suficiente como para justificar el tratamiento de estos pacientes con clonazepam. (solo un ECC con 6 pacientes tratados).

Fármacos como la rotigotina, hidroquinona, oxicodona y bromocriptina, han sido estudiados en un solo estudio cada uno, con escaso número de pacientes por lo que es insuficiente la evidencia disponible como para extraer alguna conclusión válida.

\section{Conclusión de los autores}

Algunos tratamientos han demostrado ser efectivos, como el ropinirol y la pergolida, pero otros, si bien han demostrado ser efectivos, impresionan serlo en menor medida, como la L-dopa. Sin embargo, la evidencia para todos los tratamientos disponibles hoy para el SPI es de una manera u otra, algo limitada. Los resultados más afectados por estos tratamientos fueron los movimientos periódicos de los miembros durante el sueño y la latencia del sueño. Los síntomas fueron menos afectados por estos tratamientos, a pesar de que algunos estudios demostraron que una proporción significativa de los pacientes tratados habían presentado una disminución de los síntomas y en algunos casos estos habían desaparecido. Esto es interesante porque todos los estudios incluyeron pacientes con SPI con síntomas moderados a graves, y que en su mayoría los presentaban por muchos años, algunos hasta décadas.

En general, los estudios a corto plazo demuestran poco sobre el efecto placebo. Alguno de los estudios a largo plazo con ropinirol, demostraron efectos más contundentes con placebo con duración más prolongada. Esto probablemente esté dando cuenta de una periodicidad en los síntomas del SPI. Cualquiera sea esta periodicidad produce dos consecuencias: primero, comparar tratamientos distintos en momentos distintos sería un error, y lo más importante, que los tratamientos del SPI podrían no necesitar ser continuos.

\section{Conclusión}

Los tratamientos que podrían realizarse y supervisarse directamente por el médico de familia, como el hierro o el clonazepam, no han sido efectivos o no cuentan con evidencia suficiente que demuestre beneficio alguno para el paciente que originó nuestra pregunta. La evidencia nos demuestra que, probablemente, nuestro paciente se beneficie con ropinirol o pergolida, ya que estos tratamientos han demostrado ser efectivos para el SPI. Debido a la especificidad de estos fármacos, el tipo de efectos adversos que ellos producen y su uso poco habitual en atención primaria, hacen más prudente contar con la experiencia del especialista en neurología para la elección del tratamiento más adecuado para este paciente.

\section{Referencias}

1. RA Allen et al. Restless legs: diagnostic criteria, special considerations, and epidemiology. A report from the restless legs diagnosis and epidemiology workshop at the National Institutes of Health. Sleep Medicine 2003 4: 101-119.

2. W Hening et al. Impact, diagnosis and treatment of restless legs syndrome (RLS) in a primary care population: The REST (RLS epidemiology, symptoms, and treatment) primary care study. Sleep Medicine 2004 5: 237-246.

3. http://www.jr2.ox.ac.uk/bandolier/booth/RLS/RLStreats.html, último acceso noviembre de 2005

4. http://www.clinicalanswers.nhs.uk/index.cfm?question=716, último acceso noviembre de 2005

5. http://www.jr2.ox.ac.uk/bandolier/booth/RLS/dopa.html, último acceso noviembre de 2005

6. http://www.jr2 ox ac.uk/bandolier/booth/RLS/ropin html, último acceso noviembre de 2005

7. http://www.jr2.ox.ac.uk/bandolier/booth/RLS/perg.html, último acceso noviembre de 2005

8. http://www.jr2.ox.ac.uk/bandolier/booth/RLS/carb.html, último acceso noviembre de 2005

9. http://www.jr2.ox.ac.uk/bandolier/booth/RLS/iront.html, último acceso noviembre de 2005

10. http://www.jr2.ox.ac.uk/bandolier/booth/RLS/caberg.html, último acceso noviembre de 2005

\section{Carta de lectores}

Buenos Aires, 21/07/2007.

Envío mis mas cordiales felicitaciones a los Dres. Terrasa y Yamauchi por este excelente trabajo de recopilación y por su minuciosidad científica. Más aún, quedo estimulado y sorprendido por sus comentarios y conclusiones con los que estoy plenamente identificado como medico asistencial. Sobre todo cuando el fárrago de "información científica que nos desborda..." como expresan los mismos nos introduce sesgos de resultados, con intereses que no son los nuestros. Respetando por supuesto en un todo otras intenciones, que no son las compartidas por los autores y me identifico plenamente. Nuevamente Felicitaciones por el entusiasmo que me renuevan.

Eduardo Dussaut, División Cardiología Hospital Pirovano. eduardodussaut@yahoo.com

Gracias Doctor Dussaut. Sus comentarios renuevan nuestro entusiasmo!!!

Sergio Terrasa y Gonzalo Yamauchi. 